\title{
Clinical and Microbiological Profile of Chronic Osteomyelitis Cases with Reference to Virulence Markers in Staphylococcus aureus
}

\author{
Anupama Singh ${ }^{1}$, Priyanka Paul Biswas², Aninda Sen ${ }^{3}$ \\ 1Department of Microbiology, Netaji Subhas Medical College and Hospital, Bihta, Bihar, \\ India. ${ }^{2}$ Department of Microbiology, Katihar Medical College, Katihar, Bihar, India. \\ ${ }^{3}$ Department of Microbiology, Katihar Medical College, Katihar, Bihar, India.
}

\section{ABSTRACT}

\section{BACKGROUND}

Due to increase in road traffic accidents resulting in compound fractures and also increase in the number of orthopaedic surgeons using implants, iatrogenic and chronic osteomyelitis is being encountered more frequently. The objective of this study was to describe clinical and microbiological characteristics of acute and chronic osteomyelitis with regard to virulence markers and antibiogram of isolates to prevent unnecessary morbidity and mortality.

\section{METHODS}

A total of 148 samples like pus or exudates or pieces of necrotic tissues were collected during surgery, if possible, otherwise aspirated. All samples were subjected to Gram staining and culture. Various organisms were identified by standard methods. Various virulence determinants were detected by phenotypic tests. Kirby-Bauer method was employed to perform the antimicrobial susceptibility on Mueller-Hinton agar [MHA]. For detection of methicillin-resistant Staphylococcus aureus (MRSA), MHA supplemented with $4 \% \mathrm{NaCl}$ was used.

\section{RESULTS}

Out of the 148 cases studied, 110 (74.3\%) were male and 38 (25.6\%) were female. The predominant number of osteomyelitis cases was found in the age group of 26-30 years, of these $48.5 \%$ (16/33) occurred following orthopaedic implants and $24.2 \%$ (8/33) due to post-operative wound infections. Staphylococcus aureus was the main isolate [43.9\% (58/132)]. Escherichia coli [44.4\% (4/9)] was the predominant isolate in the age group 36-40 years. The only isolate in the age group 46-50 years was Pseudomonas aeruginosa [100\% (2/2)]. Almost all the bones were involved in all age groups, but spine was mainly infected in the $46-50$ years age group. Differences in the presence of clumping factor, DNase, phosphatase, lipase, gelatinase, and presence of haemolysis on blood agar in MRSA were statistically insignificant ( $p=0.13,0.27,0.95$, $0.22,0.40,0.92$ respectively) as compared to MSSA strains.

\section{CONCLUSIONS}

MRSA strains expressing some virulence factors with multi drug resistance might play a role in pathogenesis of osteomyelitis. Therefore, novel therapeutics targeting these virulence markers, instead of conventional antibiotic therapy, as well as following new guidelines, should take place in the future that might aid in the prevention and control of MRSA infections in our hospitals.

\section{KEY WORDS}

Osteomyelitis, Methicillin Resistant Staphylococcus aureus
Corresponding Author: Priyanka Paul Biswas, Associate Professor, Department of Microbiology, Katihar Medical College, Katihar-854106, Bihar, India. E-mail: priyankaonli@yahoo.in

\section{DOI: $10.14260 /$ jemds/2020/138}

Financial or Other Competing Interests: None.

How to Cite This Article:

Singh A, Biswas PP, Sen A. Clinical and microbiological profile of chronic osteomyelitis cases with reference to virulence markers in staphylococcus aureus. J. Evolution Med. Dent. Sci. 2020;9(09):625-633, DOI: 10.14260/jemds/2020/138

Submission 11-12-2019,

Peer Review 02-02-2020,

Acceptance 11-02-2020,

Published 02-03-2020.

\section{(i)}




\section{BACKGROUND}

Infection of bone, which is called osteomyelitis, can be described as acute or chronic, haematogenous or exogenous according to the duration and source of infection. ${ }^{1}$ Osteomyelitis can become chronic and can lead to the eventual death of the bone tissue, caused by loss of blood supply to the affected bone. This occurs when pus produced within the bone, causes bone abscess which deprives bone of its blood supply. ${ }^{2}$ Treatment of chronic osteomyelitis is an eternal problem due to cavity and dead bone formation, poor blood supply, ineffective systemic antibiotic therapy with antecedent toxicity and bacterial resistance mostly due to unique biofilm formation. ${ }^{3}$ Gram-positive (GP) bacteria, especially Staphylococcus, classically causes this disease, but Gramnegative bacteria have grown in importance as causative agents. ${ }^{4,5}$ Such importance can be explained by the increasing number of orthopaedic surgeries with the use of implants and, especially, the rising number of high-energy traumas associated with open fractures, as a consequence of traffic accidents and war injuries. $6,7,8$

The dominance of Staphylococcus aureus as a causative agent of osteomyelitis may be multifactorial; such as some enzymes, surface proteins and toxins which are produced as virulence factors. ${ }^{9}$ With the emergence of mixed infections caused by gram negative bacteria secreting beta-lactamases including Extended Spectrum Beta-Lactamases (ESBL), AmpC Beta-Lactamases and Metallo-Beta-Lactamase (MBL), has led to difficulties in the treatment and eradication of infection. ${ }^{10}$ Sustained eradication of chronic osteomyelitis is difficult to achieve for several reasons, including the low levels of availability of most antibiotic agents in the chronically infected bones; the decreased metabolism of the pathogens, which are usually incorporated into a relatively impermeable glycocalyx biofilm; and the particular characteristics of the osseous environment as regards $\mathrm{pH}$ level, partial pressure of oxygen and protein concentrations. ${ }^{11}$

This study was undertaken to find out the predisposing factors leading to osteomyelitis with reference to its causative agents, evaluation of their virulence factors and determination of antimicrobial susceptibility pattern.

\section{METHODS}

\section{Study Population}

All patients who were admitted to the Department of Orthopaedics at Katihar Medical College, Katihar, Bihar, India, during the period of this project, diagnosed for osteomyelitis, and giving their consent to participate were included in the study. The project was spread over 24 months from December 2016 to November 2018. Before starting, the authors sought for and obtained ethical clearance, vide Letter No. IEC/IRB No: KMC/ IEC/ 2012-2015/ 024/ MD (micro), dated 10 $10^{\text {th }}$ November 2012, from the Institutional Ethics Committee. Written consent for voluntary participation in the study was obtained from all willing participants. In this project a total number of 148 cases were studied to ascertain the bacteriological profile of osteomyelitis, of which 122 cases showed growths of various organisms, while the remaining 26 samples were sterile. Patients with osteomyelitis of both sexes and all age groups were included in the study.

\section{Isolation and Identification}

Samples like pus or exudates or pieces of necrotic tissues were collected during surgery if possible, otherwise aspirated. Swabs were collected only when collection during surgery and aspiration was not possible. Whenever swabs were used at least two sterile cotton swabs were collected from the depths of the wound. The swabs were transferred to sterile test tubes and transported to the laboratory as soon as possible. Samples were inoculated on 5\% Blood agar (BA), MacConkey agar (MA) without crystal violet, and Mannitol salt agar (MSA) and incubated aerobically at $37^{\circ} \mathrm{C}$ for 24 hours. After incubation, the plates were examined for growth and colony morphology. If the culture showed different types of colonies, subculture was done from the different colonies to obtain pure growth. Bacterial growth on BA, MA, and MSA were processed for identification and characterization up to species level. Identification and characterization of Gram-negative organisms up to species level were done by a battery of standard tests. ${ }^{12}$

Tests for Detection of Virulence Markers with Reference to the Major Isolate (Staphylococcus aureus)

\section{Coagulase Test-}

a) Slide Test- This test detects the presence of bound coagulase enzyme. The isolated colony was emulsified in a drop of normal saline on clean microscopic slides, to make a smooth milky suspension. Similar suspension was made with positive and negative controls. A sterilized inoculating loop was dipped in undiluted plasma (derived by centrifugation of anticoagulated and pooled human blood) and stirred into the bacterial suspension. Positive and negative controls were similarly treated. Positive reaction showed coarse clumping of cocci visible to naked eye within 10 seconds.

b) Tube Coagulation Test- This test detects the free coagulase enzyme. 1-in- 6 dilution of the plasma was made in normal saline $(0.85 \% \mathrm{NaCl})$, and placed in a small, clear sterile test tube. Colonies of the test organism, positive and negative control were inoculated in separate test tubes containing the diluted plasma. The other test tube of diluted plasma was left uninoculated to detect auto coagulability of plasma. The inoculated tubes were incubated at $37^{\circ} \mathrm{C}$ in a water bath for four hours and then overnight at room temperature. Tubes were examined for clot formation at the end of $1^{\text {st }}, 2^{\text {nd }}$ and $4^{\text {th }}$ hour by tilting the tube at $90^{\circ}$. Any degree of clot formation was taken as positive. ${ }^{13}$

\section{Haemolysis}

Haemolytic activity of staphylococci was assessed on blood agar plates prepared with nutrient agar containing 5\% defibrinated sheep blood, by observation of zone of haemolysis around colonies after incubation for 24 hours at $37^{\circ} \mathrm{C} .1^{4}$

\section{Deoxyribonuclease Test}

After autoclaving and pouring of DNA media, the plates were divided into six sections by drawing lines on its bottom and sections were numbered to denote strains to be applied on 
them. Colony suspensions of the test strains were inoculated into the area marked for that particular strain. Known DNase positive and negative strains were also inoculated with each batch of tests. Plates were incubated aerobically at $37^{\circ} \mathrm{C}$ for 24 hrs. Following incubation, the plates were flooded with a few $\mathrm{ml}$ of $3.6 \% \mathrm{HCL}$ and, after standing for a few minutes, were examined against a dark background. 15

\section{Phosphatase Test}

Dehydrated phenolphthalein phosphatase agar was obtained from HiMedia Labaratories, Mumbai and medium was prepared according to manufacturer's instructions. The test organism was inoculated on the plates and incubated at $37^{\circ} \mathrm{C}$ for $24 \mathrm{hrs}$. After incubation, the plates were inverted over a few drops of ammonia solution. ${ }^{15}$ Colonies of Staphylococcus aureus turned bright pink, indicating a positive reaction.

\section{Gelatin Liquefaction Test}

Production of gelatinase enzyme was detected by gelatin liquefaction. The medium was prepared using gelatin powder as per standard procedure. A stab culture was made with the inoculum from a colony and the tubes were incubated at $37^{\circ} \mathrm{C}$. The tubes were examined daily for liquefaction of gelatin by holding the tubes at $4^{\circ} \mathrm{C}$ for 30 minutes. The test was taken as positive if gelatin remained liquid after refrigeration and the negative control remained solid. ${ }^{15}$

\section{Lipase Production}

Egg yolk agar was used for lipase production. The test organism was spot inoculated on the medium and incubated at $3^{\circ} \mathrm{C}$ for 24 to $48 \mathrm{~h}$. Positive test result was read as formation of thin iridescent pearly layer overlying the colonies and a confined opalescence in the medium, which was seen when the colonies were scraped off. 12

\section{Antimicrobial Susceptibility Testing}

Antibiotic sensitivity testing was done by modified KirbyBauer's disc diffusion test on Mueller-Hinton agar as per the Clinical and Laboratory Standards Institute (CLSI, 2013). ${ }^{16}$ For the detection of MRSA strains, MHA supplemented with $4 \%$ $\mathrm{NaCl}$ was used. Inocula was prepared and adjusted to 0.5 McFarland's standard.

\section{Screening for MRSA by Disc Diffusion Method}

The test was performed on Mueller-Hinton agar with $4 \% \mathrm{NaCl}$ for oxacillin and plain Mueller-Hinton agar for cefoxitin, 3 to 4 identical colonies of test organisms were suspended in $4 \mathrm{ml}$ peptone water and the test-tubes were incubated at $37^{\circ} \mathrm{C}$ for 2 hours, after which the turbidity was adjusted to match McFarland's standard. By using a sterile swab Mueller-Hinton agar plates were inoculated (lawn culture). Then a disc of oxacillin $1 \mu \mathrm{g}$ and cefoxitin $30 \mu \mathrm{g}$ were placed on the inoculated medium and incubated for 24 hrs at $35^{\circ} \mathrm{C}$ (CLSI 2008). ${ }^{16}$ Staphylococcus aureus ATCC 25923 was used as sensitive control.

$\begin{array}{lc}\begin{array}{l}\text { Interpretation } \\ \text { For Oxacillin }\end{array} \\ \text { Resistant } & \leq 10 \mathrm{~mm} \\ \text { Intermediate } & 11-12 \mathrm{~mm} \\ \text { Sensitive } & \geq 13 \mathrm{~mm}\end{array}$

\section{For Cefoxitin}

Resistant

Intermediate

$\leq 14 \mathrm{~mm}$

$11-17 \mathrm{~mm}$

Sensitive $\quad \geq 18 \mathrm{~mm}$

\section{Other Tests}

Serum samples were collected from patients admitted to the orthopaedic ward, on the $3^{\text {rd }}$ day of admission to ascertain the level of C-reactive protein, which was determined by latex agglutination test. Results of other tests, like total leucocyte counts and ESR were collected from the Department of Pathology. All media, stains, dyes and chemicals were obtained from HiMedia Laboratories, Mumbai, India, except for the latex agglutination kit for the estimation of C-reactive proteins, which was obtained from Span Diagnostics, Surat, India.

\section{Statistical Analysis}

Statistical analysis of data collected during the study was carried out using online software available at http://www.physics.csbsju.edu/stats/contingency_NROW_N COLUMN_form.html

\section{RESULTS}

A total of 148 cases were studied to ascertain the bacteriological profile of osteomyelitis in patients admitted in the Department of Orthopaedics of Katihar Medical College, of which 122 (82.4\%) showed growth of various organisms and remaining $26(17.6 \%)$ samples were sterile. Out of the 122 samples, 112 (91.8\%) showed monomicrobial growth and 10 (8.2\%) showed polymicrobial growth. A total of 132 organisms were isolated from the indoor patients department.

Out of the 148 cases studied, 110 (74.3\%) were male and $38(25.6 \%)$ were females. This finding was found to be statistically significant $(\mathrm{p}=0.000)$. The male to female ratio was 2.89:1. Among 148 cases, 33 cases were predominantly in the age group 26-30 years, of which 30 (90.9\%) were males and $3(9.1 \%)$ were females, which was statistically significant $(\mathrm{p}=0.000) .27$ cases were within the age-group 21-25 years, of which $19(70.3 \%)$ were males and $8(29.6 \%)$ were females. 32 cases were in the age group 16-20 years, which included 18 (56.2\%) males and 13(40.6\%) females [Table 1].

Out of the 148 cases, $34.5 \%(51 / 148)$ developed osteomyelitis after orthopaedic implants, 33.8\% (50/148) following post-traumatic injury, $16.8 \%(25 / 148)$ due to postoperative infections and $10.8 \%(16 / 148)$ due to haematogenous spread. The most commonly infected bone was found to be tibia $33.8 \%(50 / 148)$, followed by femur $23.6 \%(35 / 148)$, humerus $16.2 \%(24 / 148)$, ulna and foot $6.0 \%$ each (9/148), radius $5.4 \%(8 / 148)$, sacrum $4.0 \%$ $(6 / 148)$, and spine $3.5 \%(5 / 148)$. Hand and wrist bones (2/148) $1.3 \%$ were least involved [Table 2].

In the age group 1-5 years, (6/8) $75.0 \%$ cases were acute haematogenous and (2/8) 25.0\% were associated with orthopaedic implants. Tibia was the most commonly infected bone, (4/8; 50.0\%), followed by femur, arm and hand and wrist which were equally infected, each being $(2 / 8 ; 25.0 \%)$. In the age group 6-10 years, (7/11) $63.6 \%$ cases were acute haematogenous type with femur $(5 / 11 ; 45.5 .4 \%)$ being the main bone involved [Table 2 ]. Only $(1 / 10 ; 10.0 \%)$ case of acute 
haematogenous osteomyelitis were seen in age group 11-15 years. Most of the cases was post-traumatic $(5 / 10 ; 50.0 \%)$ and due to orthopaedic implants (4/40) $10.0 \%$. The commonly infected bones were femur and humerus $(4 / 10 ; 40.0 \%)$, each [Table 2]. Most of the osteomyelitis cases due to post traumatic injury/accidents were in two age groups respectively, 16-20 years $(19 / 32 ; 59.3 \%)$ affecting mainly the tibia $(13 / 32$; $40.6 \%)$ and in $21-25$ years $(16 / 27 ; 59.3 \%)$, with tibia $(11 / 27$; $40.7 \%$ ) being the main bone involved [Table 2].

Cases due to orthopaedic implants were seen in age group $26-30$ years $(16 / 33 ; 48.5 \%)$, followed by $(8 / 33 ; 24.2 \%)$ cases due to post-operative wound infections. The most commonly involved bone in this age group was tibia $(10 / 33 ; 30.3 \%)$, followed by femur $(7 / 33 ; 21.2 \%)$ and foot $\&$ humerus $(4 / 33$; $12.2 \%$ each) [Table 2]. In the age group $31-35$ years, $(5 / 10$; $50.0 \%)$ cases were due to post-operative infection and tibia \& femur $(3 / 10 ; 30.0 \%$ each $)$, were found to be more commonly infected [Table 2]. Similar findings were seen in the age group $36-40$ years, where most of the cases were due to postoperative infection $(4 / 9 ; 44.4 \%)$, tibia and femur $(2 / 9 ; 22.2 \%$ each) were mainly infected [Table 2].

In the age group $41-45$ years, $(4 / 6 ; 66.6 \%)$ cases were due to orthopaedic implants, and tibia $(2 / 6 ; 33.3 \%)$ was commonly infected [Table 2]. In the age group 46-50 years, $(2 / 2 ; 100 \%)$ cases were due to orthopaedic implants infecting the spine $(2 / 2 ; 100 \%)$ only [Table 2]. Of the 132 isolates, Staphylococcus aureus was the main isolate (58/132; 43.9\%), followed by Escherichia coli (25/132; 18.9\%), Klebsiella pneumoniae $(15 / 132 ; 11.4 \%)$, CONS (13/132; 9.9\%), Enterococcus spp.. (11/132; 8.3\%), Proteus mirabilis (7/132; 5.3\%) and Pseudomonas aeruginosa (5/132; 3.8\%) [Table 3].

Maximum numbers of the isolates were from the age group 26-30 years $(33 / 132 ; 25.0 \%)$, followed by $21-25$ years (27/132; $20.5 \%)$, and $16-20$ years $(24 / 132 ; 18.2 \%)$. Between the age group 1-10 years, Staphylococcus aureus (9/58; $15.5 \%$ ) was found to be the main isolate. In the age group 1115 years, Enterococcus spp. (2/11; 18.1\%), followed by Staphylococcus aureus $(2 / 58 ; 3.4 \%)$ were the main isolates. Staphylococcus aureus $(10 / 58 ; 17.2 \%)$ followed by Coagulase negative Staphylococcus $(5 / 13 ; 38.4 \%)$ were main isolates in 16-20 years age group. Proteus mirabilis $(3 / 7 ; 42.8 \%)$, followed by Enterococcus species $(3 / 11 ; 27.2 \%)$ were the most common isolates in the age group 21- 25 years. Escherichia coli $(4 / 25 ; 16.6 \%)$ was the predominant isolates in the age group $36-40$ years. The only isolate in the age group $46-50$ years was Pseudomonas aeruginosa (2/5; 40.0\%), [Table 3].

Table 4 shows the demographic details of the patients. The mean age of the patient with osteomyelitis was 22.8987 . The main comorbidity associated with osteomyelitis was diabetes mellitus $(53 / 148 ; 35.8 \%)$, followed by chronic liver disease (25/148; 16.9\%). Most of the patients were also suffering from intra-abdominal infections (49/148; 33.1\%), followed by skin and soft tissue infections $(23 / 148 ; 15.5 \%)$, urinary tract infection $(21 / 148 ; 14.2 \%)$ and arthritis $(17 / 148 ; 11.5 \%)$. The mean WBC count in osteomyelitis patients that showed growth of organisms was 8992.9 per/cubic mm whereas the mean value was 6457.94 per/cubic $\mathrm{mm}$ in cases that did not show growth of organisms. The mean CRP and ESR found in cases that showed growth of organisms were $34.104 \mu \mathrm{g} / \mathrm{ml}$ and $56.23 \mathrm{~mm}$, respectively, in $1^{\text {st }}$ hour whereas the mean CRP was $15.73 \mu \mathrm{g} / \mathrm{ml}$ and mean ESR was $33.5 \mathrm{~mm}$ in $1^{\text {st }}$ hour, in cases that did not show growth of organisms. The main clinical presentation was discharging sinus (127/148; 85.8\%), local pain $(91 / 148 ; 61.5 \%)$ and hyperaemia $(73 / 148 ; 49.3 \%)$ [Table 4].

A total of $32.7 \%$ (19/58) strains of Staphylococcus aureus were found to be MRSA, whereas $67.2 \%$ (39/58) were MSSA. Majority $(29 / 39 ; 74.4 \%)$ of the MSSA cases were found in trauma/accident cases, followed by cases with post-operative wounds $(8 / 39 ; 20.5 \%)$ and orthopaedic implants $(2 / 39$; $5.2 \%)$. On the other hand, $(9 / 19 ; 47.4 \%)$ MRSA isolates were found in patients with orthopaedic implants followed by $(7 / 19 ; 36.8 \%)$ in trauma/accident cases and $(3 / 19 ; 15.8 \%)$ in post-surgical wound infection cases [Table 4).

\begin{tabular}{|c|c|c|c|}
\hline $\begin{array}{c}\text { Age Group } \\
\text { (in years) }\end{array}$ & $\begin{array}{c}\text { Male } \\
\text { No. of Cases (\%) }\end{array}$ & $\begin{array}{c}\text { Female } \\
\text { No. of Cases (\%) }\end{array}$ & $\begin{array}{c}\text { Total } \\
\text { No. of Cases (\%) }\end{array}$ \\
\hline $1-5$ & $7(87.5)$ & $1(12.5)$ & $8(100)$ \\
\hline $6-10$ & $10(90.9)$ & $1(9.1)$ & $11(100)$ \\
\hline $11-15$ & $6(60.0)$ & $4(40.0)$ & $10(100)$ \\
\hline $16-20$ & $18(56.2)$ & $14(43.7)$ & $32(100)$ \\
\hline $21-25$ & $19(70.3)$ & $8(29.6)$ & $27(100)$ \\
\hline $26-30$ & $30(90.9)^{*}$ & $3(9.1)^{*}$ & $33(100)$ \\
\hline $31-35$ & $8(80.0)$ & $2(20.0)$ & $10(100)$ \\
\hline $36-40$ & $5(55.5)$ & $4(44.4)$ & $9(100)$ \\
\hline $41-45$ & $5(83.3)$ & $1(16.7)$ & $6(100)$ \\
\hline $46-50$ & $2(100)$ & $0(0)$ & $2(100)$ \\
\hline Total (\%) & $110(74.3){ }^{* *}$ & $38(25.6)$ & $148(100)$ \\
\hline Table 1. Age- and Gender-Wise Distribution of Osteomyelitis Cases \\
\hline \multicolumn{4}{|c|}{ Percentages calculated horizontally; ${ }^{*} \mathrm{p}=0.000 ;{ }^{* *} \mathrm{p}=0.000$} \\
\hline
\end{tabular}

\begin{tabular}{|c|c|c|c|c|c|c|c|c|c|c|c|c|c|}
\hline \multirow{3}{*}{ 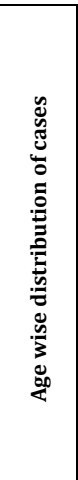 } & \multicolumn{4}{|c|}{$\begin{array}{l}\text { Pre-Disposing } \\
\text { Factors }\end{array}$} & \multicolumn{9}{|c|}{ Bones Involved } \\
\hline & 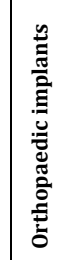 & 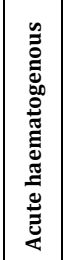 & 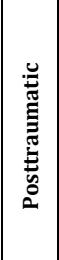 & 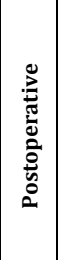 & 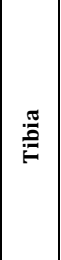 & 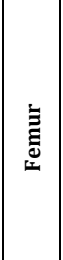 & $\stackrel{\dddot{g}}{5}$ & 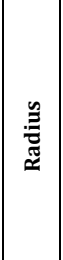 & $\ddot{\circ}$ & 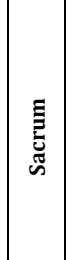 & $\stackrel{\Xi}{\ddot{m}}$ & $\stackrel{\mathscr{E}}{\Xi}$ & 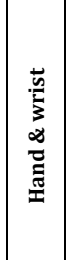 \\
\hline & $\begin{array}{l}\text { No. } \\
\text { (\%) }\end{array}$ & $\begin{array}{l}\text { No. } \\
\text { (\%) }\end{array}$ & \begin{tabular}{|l|} 
No. \\
$(\%)$
\end{tabular} & $\begin{array}{l}\text { No. } \\
(\%)\end{array}$ & $\begin{array}{l}\text { No. } \\
(\%)\end{array}$ & $\begin{array}{l}\text { No. } \\
(\%)\end{array}$ & $\begin{array}{l}\text { No. } \\
(\%)\end{array}$ & $\begin{array}{l}\text { No. } \\
(\%)\end{array}$ & $\begin{array}{l}\text { No. } \\
(\%)\end{array}$ & $\begin{array}{l}\text { No. } \\
(\%)\end{array}$ & $\begin{array}{l}\text { No. } \\
\text { (\%) }\end{array}$ & $\begin{array}{l}\text { No. } \\
(\%)\end{array}$ & $\begin{array}{l}\text { No. } \\
(\%)\end{array}$ \\
\hline $\begin{array}{c}1-5 \\
(\mathrm{~N}=8)\end{array}$ & $\begin{array}{c}2 \\
(25.0)\end{array}$ & $\begin{array}{c}6 \\
(75.0) \\
\end{array}$ & 0 & 0 & \begin{tabular}{|c|}
4 \\
$(50.0)$ \\
\end{tabular} & $\begin{array}{c}2 \\
(25.0) \\
\end{array}$ & 0 & 0 & 0 & 0 & 0 & 0 & $\begin{array}{c}2 \\
(25.0) \\
\end{array}$ \\
\hline $\begin{array}{c}6-10 \\
(\mathrm{~N}=11)\end{array}$ & $\begin{array}{c}4 \\
(36.4)\end{array}$ & $\begin{array}{c}7 \\
(63.6)\end{array}$ & 0 & 0 & $\left|\begin{array}{c}3 \\
(27.3)\end{array}\right|$ & $\begin{array}{c}5 \\
(45.5)\end{array}$ & 0 & 0 & 0 & 0 & 0 & $\left|\begin{array}{c}3 \\
(27.3)\end{array}\right|$ & 0 \\
\hline $\begin{array}{c}11-15 \\
(\mathrm{~N}=10)\end{array}$ & $\left(\begin{array}{c}4 \\
(40.0)\end{array}\right.$ & $\left(\begin{array}{c}1 \\
(10.0)\end{array}\right.$ & $\mid \begin{array}{c}5 \\
50.0)\end{array}$ & 0 & $\begin{array}{c}2 \\
(20.0)\end{array}$ & $\mid \begin{array}{c}4 \\
(40.0)\end{array}$ & 0 & 0 & 0 & 0 & 0 & $\left|\begin{array}{c}4 \\
(40.0)\end{array}\right|$ & 0 \\
\hline $\begin{array}{c}16-20 \\
(\mathrm{~N}=32)\end{array}$ & $\left(\begin{array}{c}9 \\
(28.1)\end{array}\right)$ & 0 & $\begin{array}{c}19 \\
(59.3)\end{array}$ & $\begin{array}{c}4 \\
(12.5)\end{array}$ & $\begin{array}{c}13 \\
(40.6)\end{array}$ & $\mid \begin{array}{c}6 \\
(18.8)\end{array}$ & $\begin{array}{c}3 \\
(9.4)\end{array}$ & $\begin{array}{c}4 \\
(12.5)\end{array}$ & 0 & 0 & 0 & $\begin{array}{c}6 \\
(18.8)\end{array}$ & 0 \\
\hline $\begin{array}{c}21-25 \\
(\mathrm{~N}=27)\end{array}$ & $\begin{array}{c}8 \\
(29.7)\end{array}$ & 0 & \begin{tabular}{|c|}
16 \\
$(59.3)$
\end{tabular} & $\begin{array}{c}3 \\
(11.1)\end{array}$ & $\begin{array}{c}11 \\
(40.7) \\
\end{array}$ & $\begin{array}{c}5 \\
(18.5)\end{array}$ & $\begin{array}{c}2 \\
(7.4) \\
\end{array}$ & 0 & $\begin{array}{c}3 \\
(11.1)\end{array}$ & $\begin{array}{c}2 \\
(7.4)\end{array}$ & 0 & $\begin{array}{c}4 \\
(14.8)\end{array}$ & 0 \\
\hline $\begin{array}{c}26-30 \\
(\mathrm{~N}=33)\end{array}$ & $\left.\begin{array}{c}16 \\
(48.5)\end{array}\right)$ & 0 & $\mid \begin{array}{c}9 \\
(27.3)\end{array}$ & $\begin{array}{c}8 \\
(24.2)\end{array}$ & $\begin{array}{c}10 \\
(30.3)\end{array}$ & $\left|\begin{array}{c}7 \\
(21.2)\end{array}\right|$ & $\begin{array}{c}2 \\
(6.1)\end{array}$ & $\begin{array}{c}1 \\
(3.0)\end{array}$ & $\left|\begin{array}{c}4 \\
(12.2)\end{array}\right|$ & $\mid \begin{array}{c}2 \\
(6.1)\end{array}$ & $\begin{array}{c}3 \\
(9.1)\end{array}$ & $\begin{array}{c}4 \\
(12.2)\end{array}$ & 0 \\
\hline $\begin{array}{c}31-35 \\
(\mathrm{~N}=10)\end{array}$ & $\left.\mid \begin{array}{c}2 \\
(20.0)\end{array}\right)$ & 0 & $\begin{array}{c}3 \\
(30.0)\end{array}$ & $\begin{array}{c}5 \\
(50.0)\end{array}$ & $\begin{array}{c}3 \\
(30.0)\end{array}$ & $\left|\begin{array}{c}3 \\
(30.0)\end{array}\right|$ & 0 & 0 & $\left|\begin{array}{c}1 \\
(10.0)\end{array}\right|$ & $\left(\begin{array}{c}1 \\
(10.0)\end{array}\right)$ & 0 & $\mid \begin{array}{c}2 \\
(20.0)\end{array}$ & 0 \\
\hline $\begin{array}{l}36-40 \\
(\mathrm{~N}=9)\end{array}$ & $\left|\begin{array}{c}2 \\
22.2\end{array}\right|$ & 0 & $\begin{array}{c}3 \\
(33.3)\end{array}$ & $\begin{array}{c}4 \\
(44.4)\end{array}$ & $\mid \begin{array}{c}2 \\
(22.2)\end{array}$ & $\left|\begin{array}{c}2 \\
(22.2)\end{array}\right|$ & $\begin{array}{c}1 \\
(11.1)\end{array}$ & $\left|\begin{array}{c}2 \\
(22.2)\end{array}\right|$ & $\left(\begin{array}{c}1 \\
(11.1)\end{array}\right)$ & $(11.1)$ & 0 & 0 & 0 \\
\hline $\begin{array}{l}41-45 \\
(\mathrm{~N}=6)\end{array}$ & $\begin{array}{c}4 \\
(66.6)\end{array}$ & 0 & 0 & $\begin{array}{c}1 \\
(16.7)\end{array}$ & $\begin{array}{c}2 \\
(33.3) \\
\end{array}$ & $\mid \begin{array}{c}1 \\
(16.7)\end{array}$ & $\begin{array}{c}1 \\
(16.7)\end{array}$ & $\begin{array}{c}1 \\
(16.7)\end{array}$ & 0 & 0 & 0 & 1 & 0 \\
\hline $\begin{array}{l}46-50 \\
(\mathrm{~N}=2)\end{array}$ & 0 & $\begin{array}{c}2 \\
(100)\end{array}$ & 0 & 0 & 0 & 0 & 0 & 0 & 0 & 0 & $\begin{array}{c}2 \\
(100)\end{array} \mid$ & 0 & 0 \\
\hline $\begin{array}{c}\text { Total } \\
(\mathrm{N}=148)\end{array}$ & $\left(\begin{array}{c}51 \\
(34.5)\end{array}\right.$ & $\begin{array}{c}16 \\
(10.8)\end{array}$ & $\begin{array}{c}50 \\
(33.8)\end{array}$ & $\begin{array}{c}25 \\
(16.8)\end{array}$ & $\begin{array}{c}50 \\
(33.8)\end{array}$ & $\left|\begin{array}{c}35 \\
(23.6)\end{array}\right|$ & $\begin{array}{c}9 \\
(6.0)\end{array}$ & $\begin{array}{c}8 \\
(5.4)\end{array}$ & $\begin{array}{c}9 \\
(6.0)\end{array}$ & $\begin{array}{c}6 \\
(4.0)\end{array}$ & $\begin{array}{c}5 \\
(3.5)\end{array}$ & $\begin{array}{c}24 \\
(16.2)\end{array}$ & $\mid \begin{array}{c}2 \\
(1.3)\end{array}$ \\
\hline \multicolumn{14}{|c|}{$\begin{array}{l}\text { Table 2. Age-Wise Distribution of Predisposing Factors } \\
\text { and Bones Involved in Osteomyelitis Cases }\end{array}$} \\
\hline
\end{tabular}




\begin{tabular}{|c|c|c|c|c|c|c|c|c|}
\hline \multirow{3}{*}{ 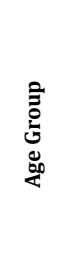 } & \multicolumn{7}{|c|}{ Organism Type } & \multirow[b]{2}{*}{ 푱 } \\
\hline & 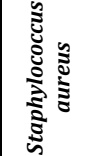 & $\sum_{0}^{n}$ & 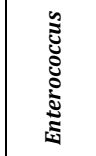 & : & 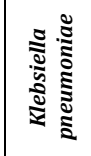 & 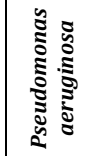 & 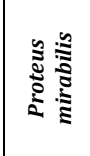 & \\
\hline & \begin{tabular}{|l|} 
No. (\%) \\
\end{tabular} & No. (\%) & No. (\%) & \begin{tabular}{|l|l|} 
No. (\%) \\
\end{tabular} & No. (\%) & No. (\%) & No. (\%) & No. (\%) \\
\hline $1-5$ & $4(6.9)$ & $3(23.0)$ & $1(9.0)$ & 0 & 0 & 0 & 0 & $8(6.0)$ \\
\hline $6-10$ & $5(8.6)$ & $3(23.0)$ & $3(27.2)$ & 0 & 0 & 0 & 0 & $11(8.3)$ \\
\hline $11-15$ & $2(3.4)$ & 0 & $2(18.1)$ & 0 & 0 & 0 & 0 & $4(3.0)$ \\
\hline $16-20$ & $\begin{array}{c}10 \\
(17.2)\end{array}$ & $\begin{array}{c}5 \\
(38.4)\end{array}$ & \begin{tabular}{|c|}
2 \\
$(18.1)$
\end{tabular} & $\begin{array}{c}3 \\
(12.0)\end{array}$ & $\begin{array}{c}3 \\
(20.0)\end{array}$ & 0 & $\begin{array}{c}1 \\
(14.3)\end{array}$ & $\begin{array}{c}24 \\
(18.1)\end{array}$ \\
\hline $21-25$ & $\begin{array}{c}11 \\
(18.9)\end{array}$ & $\begin{array}{c}1 \\
(7.7)\end{array}$ & $\begin{array}{c}3 \\
(27.2)\end{array}$ & $\begin{array}{c}5 \\
(20.0)\end{array}$ & $\begin{array}{c}4 \\
(26.6)\end{array}$ & 0 & $\begin{array}{c}3 \\
(42.8) \\
\end{array}$ & $\begin{array}{c}27 \\
(20.4)\end{array}$ \\
\hline $26-30$ & $\begin{array}{c}15 \\
(25.8) \\
\end{array}$ & $\begin{array}{c}1 \\
(7.7)\end{array}$ & 0 & $\begin{array}{c}7 \\
(28.0) \\
\end{array}$ & $\begin{array}{c}4 \\
(26.6) \\
\end{array}$ & $\begin{array}{c}3 \\
(60.0) \\
\end{array}$ & $\begin{array}{c}3 \\
(42.8) \\
\end{array}$ & $\begin{array}{c}33 \\
(25.0) \\
\end{array}$ \\
\hline $31-35$ & $5(8.6)$ & 0 & 0 & $4(16.0)$ & $1(6.6)$ & 0 & 0 & $10(7.6)$ \\
\hline $36-40$ & $3(5.1)$ & 0 & 0 & $4(16.0)$ & $2(13.3)$ & 0 & 0 & $9(6.8)$ \\
\hline $41-45$ & $3(5.2)$ & 0 & 0 & $2(8.0)$ & $1(6.6)$ & 0 & 0 & $6(4.5)$ \\
\hline $46-50$ & 0 & 0 & 0 & 0 & 0 & $2(40.0)$ & 0 & $2(1.5)$ \\
\hline Total & 58 & 13 & 11 & 25 & 15 & 5 & 7 & 132 \\
\hline
\end{tabular}

\begin{tabular}{|c|c|c|c|}
\hline Characteristics & \multicolumn{3}{|c|}{ Values } \\
\hline \multicolumn{4}{|c|}{ Age; $\mathrm{N}=148$} \\
\hline Mean & \multicolumn{3}{|c|}{22.898} \\
\hline Median & \multicolumn{3}{|c|}{22.351} \\
\hline Comorbidity; $\mathrm{N}=148$ & \multicolumn{3}{|c|}{ No. (\%) } \\
\hline Diabetes mellitus & \multicolumn{3}{|c|}{$\frac{1 \text { No. (20) }}{53(35.8)}$} \\
\hline Liver disease & \multicolumn{3}{|c|}{$21(14.2)$} \\
\hline Chronic kidney disease & \multicolumn{3}{|c|}{$25(16.9)$} \\
\hline Preceding or co-infection; N=148 & \multicolumn{3}{|c|}{ No. $(\%)$} \\
\hline Urinary tract infection & \multirow{2}{*}{\multicolumn{3}{|c|}{$\begin{array}{l}21(14.2) \\
49(33.1)\end{array}$}} \\
\hline Intra-abdominal infection & & & \\
\hline Skin and soft tissue infection & \multicolumn{3}{|c|}{$23(155)$} \\
\hline Arthritis & \multicolumn{3}{|c|}{$17(11.5)$} \\
\hline \multicolumn{4}{|c|}{ Laboratory findings in cases that showed growth of organisms; $N=122$} \\
\hline WBC (per cubic mm, mean) & \multicolumn{3}{|c|}{\begin{tabular}{|r|}
8992.9 \\
\end{tabular}} \\
\hline $\mathrm{CRP}(\mu \mathrm{g} / \mathrm{ml}, \mathrm{mean})$ & \multicolumn{3}{|c|}{34.104} \\
\hline ESR (mm in 1st hour, mean) & \multicolumn{3}{|c|}{56.231} \\
\hline \multicolumn{4}{|c|}{ Laboratory findings in cases that did not show growth of organisms; $N=26$} \\
\hline WBC (per cubic mm, mean) & \multirow{2}{*}{\multicolumn{3}{|c|}{\begin{tabular}{|c|}
6457.94 \\
1573
\end{tabular}}} \\
\hline $\mathrm{CRP}(\mu \mathrm{g} / \mathrm{ml}, \mathrm{mean})$ & & 15.733 & \\
\hline ESR (mm in 1st hour, mean) & \multicolumn{3}{|c|}{$\begin{array}{c}15.733 \\
33.5\end{array}$} \\
\hline Presentation of infection; N=148 & \multicolumn{3}{|c|}{ No. (\%) } \\
\hline Fever & \multicolumn{3}{|c|}{$31(20.9)$} \\
\hline Back pain & \multicolumn{3}{|c|}{$5(3.4)$} \\
\hline Discharging sinus & \multicolumn{3}{|c|}{$127(85.8)$} \\
\hline Local pain & & $91(61.5)$ & \\
\hline Hyperaemia & & $73(49.3)$ & \\
\hline Functional Disability & & $7(4.7)$ & \\
\hline Characteristics (MRSA/MSSA); $\mathrm{N}=58$ & & No. (\%) & \\
\hline MRSA isolates & & $19(32.7)$ & \\
\hline MSSA isolates & & $39(67.2)$ & \\
\hline $\begin{array}{l}\text { Predisposing factors in MRSA isolates; } \\
\mathrm{N}=19\end{array}$ & & No. (\%) & \\
\hline Trauma/accidents & & $7(36.8)$ & \\
\hline Orthopaedic implants & & $9(47.4)$ & \\
\hline Post-surgical wounds & & $3(15.8)$ & \\
\hline $\begin{array}{l}\text { Predisposing factors in MSSA isolates; } \\
\mathrm{N}=39\end{array}$ & & No. (\%) & \\
\hline Trauma/accidents & & $29(74.4)$ & \\
\hline Orthopaedic implants & & $2(5.2)$ & \\
\hline Post-surgical wounds & & $\frac{2(3.2)}{8(20.5)}$ & \\
\hline & MRSA, $\mathrm{N}=19$ & MSSA, $\mathrm{N}=39$ & \\
\hline Virulence markers & \begin{tabular}{|c|} 
No. (\%) \\
\end{tabular} & No. (\%) & P-value \\
\hline Slide coagulase/clumping factor & $17(89.5)$ & $37(94.9)$ & 0.446 \\
\hline Tube coagulase/free coagulase & $19(100)$ & $39(100)$ & \\
\hline DNase & $14(73.7)$ & $23(58.9)$ & 0.274 \\
\hline Phosphatase & $14(73.7)$ & $29(74.4)$ & 0.956 \\
\hline Gelatinase & $16(84.2)$ & $27(69.2)$ & 0.221 \\
\hline Lipase & $10(52.6)$ & $16(41.0)$ & 0.404 \\
\hline Haemolysis on blood agar & $10(52.6)$ & $20(51.3)$ & 0.923 \\
\hline $\begin{array}{l}\text { Table 4. Demographic Details } \\
\text { Virulence Markers in M }\end{array}$ & of Patients & $\begin{array}{l}\text { nd Distribu } \\
\text { SA Isolates }\end{array}$ & on of \\
\hline
\end{tabular}

Table 4 also shows the result of various phenotypic tests done for detection as well as of identification of virulence factors of methicillin-resistant and methicillin-sensitive
Staphylococcus aureus. 89.5\% (17/19) of the MRSA strains tested were positive for clumping factor in the slide coagulase test, as compared to, $94.8 \%$ (37/39) of MSSA strains. All the MRSA and MSSA strains tested were positive for free coagulase in the tube coagulase test. However, 73.7\% (14/19) MRSA strains tested were positive for production of DNase as compared to $58.9 \%(23 / 39)$ of the MSSA strains. This finding was statistically insignificant $(\mathrm{p}=0.274)$. The expression of gelatinase and lipase were seen in $84.2 \%(16 / 19)$ and $52.6 \%$ (10/19) of MRSA as compared to $69.2 \%$ (27/39) and $41.0 \%$ $(16 / 39)$ of MSSA strains. These findings were also statistically insignificant $(\mathrm{p}=0.446$ and $\mathrm{p}=0.404)$. Of the MRSA, $73.7 \%$ $(14 / 19)$ were found to produce phosphatase as compared to $74.4 \%$ (29/39) of the MSSA strains, which was also statistically insignificant $(p=0.956) .52 .6 \%(10 / 19)$ MRSA strains produced beta haemolysis whereas the production was slightly lower in the MSSA strains, $51.3 \%$ (20/39), the results being statistically insignificant $(\mathrm{p}=0.923)$.

Table 5 shows the antibiotic susceptibility pattern of MRSA, MSSA and CONS. Maximum resistance in case of MSSA was seen with the following antibiotics: amoxicillin followed by cephalexin $(100 \%)$ and ciprofloxacin $(76.9 \%)$, In case of MRSA, resistance was seen with amoxicillin (100\%) followed by cephalexin, netilmicin and ciprofloxacin (68.4\%, each). Of the MRSA, $26.3 \%$ and $5.3 \%$ strains were resistant to vancomycin and linezolid respectively, by disc diffusion method. In case of CONS, $100 \%$ resistance was seen with amoxicillin followed by $76.9 \%$ resistance with cephalexin.

\begin{tabular}{|c|c|c|c|c|c|c|}
\hline \multirow[t]{2}{*}{ Antibiotics } & \multicolumn{2}{|c|}{$\begin{array}{c}\text { Methicillin Sensitive } \\
\text { S. aureus (MSSA); } \\
\mathrm{N}=39\end{array}$} & \multicolumn{2}{|c|}{\begin{tabular}{|c} 
Methicillin \\
Resistant \\
S. aureus (MRSA); \\
$\mathrm{N}=19$
\end{tabular}} & \multicolumn{2}{|c|}{ CONS; $N=13$} \\
\hline & \begin{tabular}{|c|} 
Sensitive \\
No. (\%)
\end{tabular} & $\begin{array}{c}\text { Resistant } \\
\text { No. (\%) }\end{array}$ & \begin{tabular}{|} 
Sensitive \\
No. (\%)
\end{tabular} & $\begin{array}{l}\text { Resistant } \\
\text { No. (\%) }\end{array}$ & $\begin{array}{c}\text { Sensitive } \\
\text { No. (\%) }\end{array}$ & $\begin{array}{c}\text { Resistant } \\
\text { No. (\%) }\end{array}$ \\
\hline Amoxycillin & 0 & $39(100)$ & 0 & $19(100)$ & 0 & $13(100)$ \\
\hline Cephalexin & $9(23.0)$ & $30(76.9)$ & $6(31.6)$ & $13(68.4)$ & $3(23.0)$ & $10(76.9)$ \\
\hline Netilmicin & $12(30.7)$ & $27(69.2)$ & $6(31.6)$ & $13(68.4)$ & $7(53.8)$ & $6(46.2)$ \\
\hline Ciprofloxacin & $9(23.0)$ & $30(76.9)$ & $6(31.6)$ & $13(68.4)$ & $8(61.5)$ & $5(38.5)$ \\
\hline Erythromycin & $17(43.6)$ & $22(56.4)$ & $7(36.8)$ & $12(63.2)$ & $6(46.2)$ & $7(53.8)$ \\
\hline Amikacin & $30(76.9)$ & $9(23.0)$ & $6(31.6)$ & $13(68.4)$ & $10(76.9)$ & $3(23.0)$ \\
\hline Vancomycin & $39(100)$ & 0 & $14(73.7)$ & $5(26.3)$ & $10(76.9)$ & $3(23.0)$ \\
\hline Linezolid & $39(100)$ & 0 & 18(94.7) & $1(5.3)$ & $12(92.3)$ & $1(1.0)$ \\
\hline Clindamycin & $30(76.9)$ & $9(23.0)$ & $15(78.9)$ & $4(21.1)$ & $11(84.6)$ & $2(15.4)$ \\
\hline Gentamicin & $30(76.9)$ & $9(23.0)$ & $7(36.8)$ & $12(63.2)$ & $11(84.6)$ & $2(40.0)$ \\
\hline \multicolumn{7}{|c|}{$\begin{array}{c}\text { Table 5. Antibiotic Sensitivity/Resistant Pattern of } \\
\text { Staphylococcus Species }\end{array}$} \\
\hline
\end{tabular}

\section{DISCUSSION}

Osteomyelitis is one of the most inconveniencing diseases among most of the developing countries like India. ${ }^{17}$ Chronic osteomyelitis is notoriously resistant to treatment and requires aggressive surgical debridement in addition to antibiotic therapy. ${ }^{18}$ Chronic osteomyelitis may require antimicrobial therapy for months to years, sometimes with antibiotics that are invaluable for the hospital environment, such as glycopeptides and carbapenems. ${ }^{19}$ This situation makes the accurate identification of the pathogen an absolute cornerstone of antimicrobial therapy. ${ }^{20}$ Widespread use of antibiotics has altered etiological pattern of infections and their antibiotic susceptibility. Hence continuous monitoring of 
susceptibility pattern needs to be carried out in individual setting so as to detect the true burden of antibiotic resistance among organisms and prevent their further emergence by judicious use of drugs. ${ }^{20}$

A total of 148 cases were studied to ascertain the bacteriological profile of Osteomyelitis in patients admitted in the Department of Orthopaedics of Katihar Medical College, of which 122 (82.4\%) showed growth of various organisms and remaining $26(17.6 \%)$ samples were sterile. Out of the 122 samples, 112 (91.8\%) showed monomicrobial growth and 10 (8.2\%) showed polymicrobial growth. A total of 132 organisms were isolated from indoor patient department. Wadekar D. M. et al, 2014, reported similar findings where $87.0 \%$ samples were found to be culture positive whereas $13.0 \%$ samples were culture negative; monomicrobial growth was seen in $67.0 \%$ and polymicrobial growth in $20.0 \%$ cases. Collection of specimens before the administration of antibiotics, use of proper transport media and other factors play a role in incidence of positive culture. ${ }^{21}$

Out of the 148 cases studied, 110 (74.3\%) were male and $38(25.6 \%)$ were females. This finding was found to be statistically significant $(\mathrm{p}=0.000)$. The male to female ratio was 2.89:1. Among the 148 cases, 33 cases were predominantly of the age group 26-30 years, of which 30 (90.9\%) were males and 3 (9.1\%) females, which was again a statistically significant $(\mathrm{p}=0.000)$ finding. Authors also reported a higher incidence of osteomyelitis in male than in females, with the ratio of 2.7:1. The predominance of male patients may point towards gender bias present in the society. This can also be attributed to more exposure to trauma in males. Similar findings where noted where the incidence of osteomyelitis was $84.0 \%$ in males and $16.0 \%$ in females, male to female ratio being 5.25:1.21,22 Other reports showed highest infection rate of osteomyelitis cases between 30-40 years (29.0\%) followed by $20-30$ years $(23.0 \%), 10-20$ years $(17.0 \%), 40-50$ years (15.0\%), 1-10 (8.0\%) and $50 \&$ above (7.0\%). ${ }^{17}$

Of the 148 orthopaedic cases, $34.5 \%(51 / 148)$ were due to orthopaedic implants followed by $33.8 \%(50 / 148)$ of posttraumatic injury, $14.2 \%(25 / 148)$ due to post-operative infections and $10.8 \%(16 / 148)$ due to acute haematogenous type of infection. On the other hand, other authors reported the common predisposing factor for osteomyelitis to be trauma (44.0\%) which was followed by postsurgical infections (23.0\%) and orthopaedic implants (21.0\%). ${ }^{10}$ The most commonly infected bone was found to be tibia (48/148) $32.4 \%$ which was followed by femur $22.9 \%$ (34/148), humerus $16.2 \%(24 / 148)$, ulna \& foot being $6.0 \%$ (9/148) respectively and radius $5.4 \%(8 / 148) \&$ sacrum $4.0 \%(6 / 148)$. Spine $3.5 \%$ (5/148) followed by arm $2.0 \%(3 / 148)$ and hand \& wrist bones $1.3 \%(2 / 148)$ were least involved. Other studies also found that tibia was the commonest bone affected by osteomyelitis (44.0\%), followed by femur (40.8\%), metatarsals (4.0\%), while fibula was the least affected $(0.8 \%))^{23}$ However, in contrast other authors found highest incidence of osteomyelitis in femur (48\%), followed by tibia $(23 \%)$ and humerus (9\%). ${ }^{10}$

Our study results showed that, of the age group 1-5 years, $75.0 \%(6 / 8)$ cases were acute haematogenous and $25.0 \%$ $(2 / 8)$ were associated with orthopaedic implants. Bones like tibia, femur, arm and hand \& wrist were equally infected, each being $25.0 \%$ (2/8). Acute haematogenous osteomyelitis refers to infection of bone resulting from bacteria in the bloodstream. This is seen most often in children, with initial infection thought to occur in the richly vascularised metaphyseal region. Children are thought to experience frequent episodes of bacteraemia, often with no apparent symptoms, leading to seeding and development of osteomyelitis. In children, osteomyelitis affects long bones in legs and upper arms, including femur and humerus. However, the incidence in our study was found to be low in age group 1-10 years (1.6\%) which reflects on better management of delivery in institutional care, improved standard of living and early administration of broad-spectrum antibiotics. ${ }^{24}$

Most of the osteomyelitis cases due to post traumatic injury/accidents were in two age groups, 16-20 years being $59.3 \%$ (19/32) affecting tibia 40.6\% (13/32) more commonly and in $21-25$ years, being $59.3 \%(16 / 27)$ cases, again with tibia $40.7 \%(11 / 27)$ being the main bone involved. Other reports shows similar findings where majority of the osteomyelitis cases were in the 16-30 years age group, which is attributable to the greater likelihood of trauma and compound fractures at this age group. ${ }^{19}$ Other authors reported highest infection rate of osteomyelitis cases between $30-40$ years $(29.0 \%)$ followed by $20-30$ years $(23.0 \%), 10-20$ years $(17.0 \%), 40-50$ years $(15.0 \%), 1-10(8.0 \%)$ and $50 \&$ above $(7.0 \%) .{ }^{17}$

The causative agent of osteomyelitis is often associated with mode of infection and age of the patient. Maximum numbers of the isolates were from the age group 26- 30 years $25.0 \%$ (33/132), followed by $21-25$ years $20.5 \%$ (27/132) and 16-20 years $18.2 \%$ (24/132). Our study showed the most common causative agent was Staphylococcus aureus $43.9 \%$ (58/132), which is consistent with studies conducted by others. ${ }^{19,25}$ Staphylococcus aureus is by far the most commonly involved, because organism elaborates a range of extracellular and cell-associated factors contributing to its virulence. Its ability to invade the tissues and formation of biofilms explain the persistent bone infection caused by this organism. ${ }^{26}$

Among other organisms isolated are Escherichia coli $18.9 \%$ (25/132), Klebsiella pneumoniae 11.4\% (15/132), CONS 9.9\% (13/132), Enterococcus spp. 8.3\% (11/132), Proteus mirabilis 5.3\% (7/132) and Pseudomonas aeruginosa $3.8 \%$ (5/132).This is consistent with studies conducted by others. ${ }^{10,22}$ Although gram-negative bacilli represent a minor portion of all the pathological agents isolated in osteomyelitis cases, they are of major clinical importance due to the peculiarities of antimicrobial susceptibility pattern and due to the co-morbidities generally affecting those patients. Enteric gram-negative bacilli are amongst the common causative agents as many orthopaedic patients are bedridden for prolonged periods. ${ }^{27}$

In our study, results showed that within the age group 110 years, Staphylococcus aureus $47.4 \%$ (9/19) was found to be the main isolate. In the age group $11-15$ years, Staphylococcus aureus and Enterococcus spp.. were the main isolates, being $50.0 \%$ (2/4) each. Similarly, Staphylococcus aureus 41.7\% $(10 / 24)$ followed by Coagulase negative Staphylococcus (CONS) being $20.8 \%(5 / 24)$ were the main isolates in $16-20$ years age group. Staphylococcus aureus 40.7\% (11/27) followed by Escherichia coli 18.5\% (5/27) and Klebsiella 
pneumoniae $14.8 \%$ (4/27) were the main isolates in the age group 21-25 years. Escherichia coli $44.4 \%$ (4/9) was the predominant isolate in the age group 36-40 years. Authors reported similar findings, where Staphylococcus aureus was the commonest organism isolated in children, while in adults, Gram negative organisms were commonly isolated because infection is commonly secondary to the contiguous focus of infection. ${ }^{19}$

Majority of the MSSA isolates (74.4\%) were found in trauma/accident cases. On the other hand, $47.4 \%$ MRSA isolates were found in patients with orthopaedic implants. Petty et al, 1995 \& Von Eiff et al, 2002 reported Staphylococcus aureus to be the common cause of metal-biomaterial, bonejoint infection and Staphylococcus epidermidis to be the cause of polymer-associated implant infections. ${ }^{28,29}$ It has been reported that MRSA can disseminate to bones by the haematogenous route and can also adhere to prosthetic devices, leading to biofilm formation in case of osteomyelitis. ${ }^{30}$

Staphylococcus aureus can adhere to and become internalized by a variety of host cells, including osteoblasts. Uptake of organisms is promoted by fibronectin binding proteins, once internalized bacteria can escape the phagosomes and cause necrosis. ${ }^{31}$ All MRSA and MSSA isolates were investigated for some extracellular virulence factors, such as protease, lipase and haemolysin. The slide coagulase test which was done for detection of clumping factor was positive in $57.9 \%(11 / 19)$ of MRSA strains as compared to $76.9 \%$ (30/39) of MSSA strains. This finding was found to be statistically insignificant $(p=0.135)$. Similar findings were reported by other authors where they found MRSA was a weak producer of clumping (slide coagulase) factor, which in turn explains why only $60 \%$ of the isolates gave positive results in the slide agglutination test. Therefore, the tube coagulase method is more reliable than slide coagulase method in the diagnosis of MRSA. ${ }^{32}$ Coagulase is an extracellular protein that exists in free and bound forms and bind prothrombin in a ratio of 1:1 to form staphylothrombin. This binding reaction, catalyses plasma clotting. Such clots may provide protection to the bacterium, which may also become coated with fibrin, thereby inhibiting phagocytosis. Since clot formation is an important pathophysiological process in osteomyelitis, it is tempting to speculate that coagulase may be an important virulence factor for Staphylococcus aureus in this condition. ${ }^{31}$ However it is possible that a thin layer of fibrin may inhibit phagocytosis for long enough to allow organisms to become established on bone or synovium, following which other protective mechanisms may come into play. ${ }^{31}$

$73.7 \%(14 / 19)$ MRSA strains tested in our study were positive for production of DNase whereas only $58.9 \%$ (23/39) MSSA were DNase positive. Even, this finding was statistically insignificant $(p=0.274)$. Others reported that, $14 \%$ of the MRSA isolates were negative for DNase. ${ }^{32}$ Another study by Kateete David P et al., gives 25\% negativity for DNase test. ${ }^{33}$

$52.6 \%(10 / 19)$ MRSA were found to produce lipase as compared to $41.0 \%(16 / 39)$ MSSA strains. Expressions of lipase play a strong role in pathogenesis of the disease. On the other hand, Lacey and co-workers discussed their experience in which they found that MRSA lacked lipase activity. Enzyme lipase has been suggested to be an important determinant in evading host defences. The host produces a range of fatty acids and lipids that can attack bacteria in response to infection and it has been postulated that Staphylococcus aureus lipases counteract these molecules. To date no deletion mutants of lipases have been studied in animal models, so it is difficult to ascribe to these proteins, relevance to the pathology of bone and joint infections. ${ }^{34}$

Investigations were carried out to find out the role of exotoxins, namely $(\alpha, \beta$ and $\gamma$ ) as virulence factors in Staphylococcus aureus induced bone pathology. $\alpha$-toxin, a potent membrane damaging toxin, is responsible for tissue invasion. Our study showed that only $31.6 \%(6 / 19)$ MRSA secreted $\alpha$-haemolysins as compared to $28.2 \%(11 / 39)$ MSSA strains. Moreover, $52.6 \%$ (10/19) MRSA strains produced $\beta$ haemolysins whereas the production was slightly lower at $51.3 \%$ (20/39) in the MSSA strains. Authors say that most strains of Staphylococcus aureus isolated from humans, do not produce $\beta$-toxin probably because of phage conversion, and so this toxin is unlikely to be important in bone and joint pathology. ${ }^{31}$

Our results showed that $15.8 \%$ (3/19) MRSA expressed $\gamma$ haemolysin as compared to $20.2 \%(8 / 39)$ MSSA strains. Previous reports say that antibodies to $\gamma$ - haemolysin have been assessed as a method of diagnosis of bone and joint infection, but it is unclear what significance should be attached to this in terms of pathogenesis. ${ }^{35}$

Majority of the MSSA (100\%) showed resistance to amoxicillin, followed by cephalexin and ciprofloxacin $(76.9 \%$ each); whereas in case of MRSA resistance was seen with amoxicillin (100\%), followed by cephalexin, netilmicin, and ciprofloxacin being $68.4 \%$ each. Of the MRSA, $26.3 \%$ and $5.3 \%$ strains were resistant to vancomycin and linezolid respectively, by disc diffusion method. In case of CONS $100 \%$ resistance was seen with amoxicillin followed by $76.9 \%$ resistance with cephalexin. Ali M. et al, 2014, reported that all the MSSA strains were susceptible to vancomycin, gentamicin, teicoplanin, ciprofloxacin and linezolid. The authors also reported that the MRSA strains were sensitive to vancomycin and linezolid. Kaur J et al, 2008, reported that 27.9\% (39/43) strains were MRSA, which were found to produce betalactamase and were multi-drug resistant seen in $55.8 \%$ of the strains. ${ }^{19}$ In a study by Suguneswari G. et al, 2013, the MRSA isolates showed resistance to commonly used antibiotics like cefepime (100\%), erythromycin (90.9\%), tetracycline (90.9\%), co-trimoxazole (90.9\%), piperacillin/tazobactam (81.8\%), ciprofloxacin (72.7\%) and levofloxacin (54.5\%). ${ }^{17}$ However, all the MRSA strains showed $100 \%$ sensitivity to vancomycin and $91.6 \%$ sensitivity to levofloxacin. It is quite clear from the studies that has been conducted so far, as well as from the present study, that MRSA strains are becoming alarming because of their increased resistance towards antibiotics like amikacin, netilmicin, and to a lesser extent to vancomycin and linezolid, that leaves the clinicians with less choice to use as appropriate drugs for treatment of chronic osteomyelitis.

\section{CONCLUSIONS}

Although future appears bleak for the antibiotic treatment of Staphylococcus aureus infections, our advances in 
understanding the mechanisms by which this organism causes bone and joint infection will no doubt lead to the identification of candidate therapeutic targets of both bacterial and host origin. Thus, alternatives to antibiotic treatment are likely to be available in the not too distant future.

\section{REFERENCES}

[1] Sax H, Lew D. Osteomyelitis. Curr Infect Dis Rep 1999;1(3):261-6.

[2] Twaij JH, AL-Najar AR, AL-Hashemi EH. Clinical and bacteriological analysis of osteomyelitis. Iraqi J Comm Med 2001;14(2):201-5.

[3] Stewart PS. Mechanisms of antibiotic resistance in bacterial biofilms. Int J Med Microbiol 2002;292(2):10713.

[4] Berbari EF, Steckelberg JM, Osmon DR. Osteomyelitis. In: Mandell G, edr. Principles and practice of infectious diseases. Philadelphia: Elsevier 2005: p. 1322.

[5] Peleg AY, Hooper DC. Hospital-acquired infections due to gram-negative bacteria. $\mathrm{N}$ Engl J Med 2010;362(19):1804-13.

[6] Del Pozo JL, Patel R. Infection associated with prosthetic joints. N Engl J Med 2009;361(8):787-94.

[7] Johnson EN, Burns TC, Hayda RA, et al. Infectious complications of open type III tibial fractures among combat casualties. Clin Infect Dis 2007;45(4):409-15.

[8] Lima AL, Oliveira PR, Paula AP. Acinetobacter infection. N Engl J Med 2008;358(26):2846.

[9] Chambers HF. The changing epidemiology of Staphylococcus aureus. Emerg Infect Dis 2001;7(2):17882.

[10] Wadekar MD, Anuradha K, Venkatesha D. Chronic osteomyelitis: aetiology and antibiotic susceptibility pattern. Int Journal of Recent Trends in Science and Technology 2014;9(3):337-40.

[11] Senneville E, Melliez H, Beltrand E, et al. Culture of percutaneous bone biopsy specimens for diagnosis of diabetic foot osteomyelitis: concordance with ulcer swab cultures. Clinical Infectious Diseases 2006;42(1):57-62.

[12] Winn WJ, Allen S, Janda W, et al. Koneman's Colour Atlas and Textbook of Diagnostic Microbiology. $6^{\text {th }}$ edn. Philadelphia: Lippincott Williams \& Wilkins 2006.

[13] Baird D. Staphylococcus: cluster forming Gram-positive cocci. In: Colle JG, Fraser AG, Marmion BP, et al. eds. Mackie and McCartney Practical Medical Microbiology. 14th edn. New Delhi: Churchill Livingstone 2006: p. 24526.

[14] El-Gayar, Aboulwafa M, Mona, et al. Virulence characters of some methicillin resistant Staphylococcus aureus isolate. Arc of Clin Microbiol 2014;5(4):1-14.

[15] Collee JG, Miles RS, Watt B. Test for identification of bacteria. In: Collee JG, Fraser AG, Marmion BP, et al. eds. Mackie \& McCartney Practical Medical Microbiology. 14th edn. New Delhi: Churchill Livingstone 2006: p. 131-50.

[16] Clinical and Laboratory Standards Institute: Performance Standards for Antimicrobial Susceptibility testing, 23 ${ }^{\text {rd }}$
Informational Supplement, CLSI document M100-S23, Wayne, PA, 2013;33(1).

[17] Suguneswari G, Singh AH, Basu R. Bacteriological profile of osteomyelitis in a tertiary care hospital at Visakhapatnam, Andhra Pradesh. Int J Cur Res Rev 2013;5(20):52-8.

[18] Mackowiak PA, Jones SR, Smith JW. Diagnostic value of sinus-tract cultures in chronic osteomyelitis. JAMA 1978;239(26):2772-5.

[19] Kaur J, Gulati VL, Aggarwal A, et al. Bacteriological profile of osteomyelitis with special reference to Staphylococcus aureus. Indian J for the Practicing Doctors 2008;4(6):1-9.

[20] Zuluaga AF, Galvis W, Jaimes F, et al. Lack of microbiological concordance between bone and nonbone specimens in chronic osteomyelitis: an observational study. BMC Infect Dis 2002;2:1-7.

[21] Ali M, Kumar R. Evaluation of bacteriological profile of chronic osteomyelitis in a tertiary care hospital. Int J Scientific Research 2014;(3):383-5.

[22] Mohsien RA, Al-Mohammedawi M, Yahya AGI, et al. Virulence factors enhancing microbial infection in chronic osteomyelitis and antibiotic susceptibility pattern. Am J of Med Case Rep 2014;2(6):126-32.

[23] Khatoon R, Khan SA, Jahan N. Antibiotic resistance pattern among aerobic bacterial isolates from osteomyelitis cases attending a Tertiary care hospital of North India with special reference to ESBL, AmpC, MBL and MRSA production. Int J Res Med Sci 2017;5(2):482-90.

[24] Conrad DA. Acute haematogenous osteomyelitis. Paediatrics in Review 2010;31(11):464-71.

[25] Agarwal S, Zahid M, Sherwani MKA, et al. Comparison of the results of sinus track culture and sequestrum culture in chronic osteomyelitis. Acta Orthop Belg 2005;71(2):209-12.

[26] Lew DP, Waldvogel FA. Osteomyelitis. Lancet 2004;364(9431):369-79.

[27] De Carvalho VC, De Oliveira PRD, Dal-Paz K, et al. Gramnegative osteomyelitis: clinical and microbiological profile. Braz J Infect Dis 2012;16(1):63-7.

[28] Petty W, Spanier S, Shuster JJ, et al. The influence of skeletal implants on incidence of infection. Experiments in a canine model. J Bone Joint Surg Am 1985;67(8):123644.

[29] Von Eiff C, Peters G, Heilmann C. Pathogenesis of infections due to coagulase negative staphylococci. Lancet Infect Dis 2002;2(11):677-85.

[30] Waness A. Revisiting methicillin-resistant Staphylococcus aureus infections. Journal of Global Infectious Diseases 2010;2(1):49-56.

[31] Nair SP, Williams RJ, Henderson B. Advances in our understanding of bone and joint pathology caused by Staphylocccus aureus infection. British J of Rheumatology 2000;39(8):821-34.

[32] Monica C. District Laboratory Practices in Tropical Countries. Part-2. Second edition. Cambridge University Press 2006: p. 67.

[33] Kateete DP, Kimani CN, Katabazi FA, et al. Identification of Staphylococcus aureus: DNase and Mannitol salt agar improve the efficiency of the tube coagulase test. Annals of Clinical Microbiology and Antimicrobials 2010;9:23. 
[34] Lacey RW, Barr KW, Barr V, et al. Properties of methicillinresistant Staphylococcus aureus colonizing patients in a burns unit. Hospital Infections 1986;7(2):137-48.
[35] Taylor AG, Bernheiner AW. Further characterization of staphylococcal gamma-haemolysin. Infect Immun 1974;10(1):54-9. 